Historic, Archive Document

Do not assume content reflects current scientific knowledge, policies, or practices. 



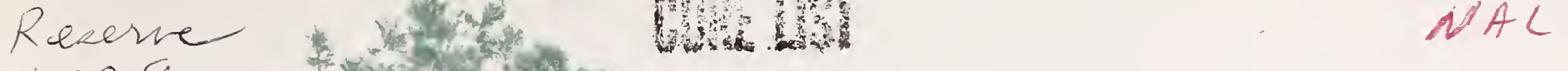

A99.9 * of

F 76324

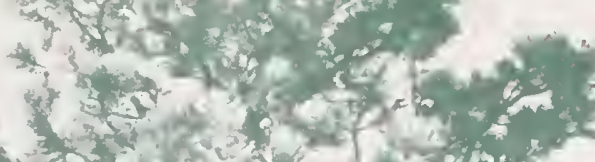
1.

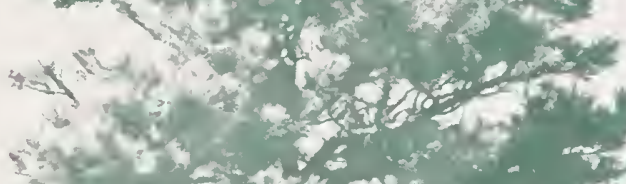

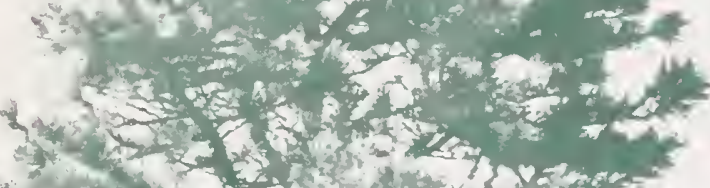

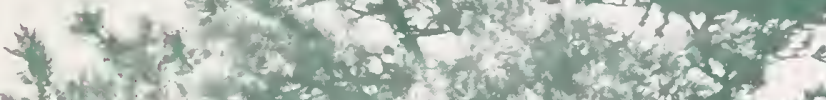

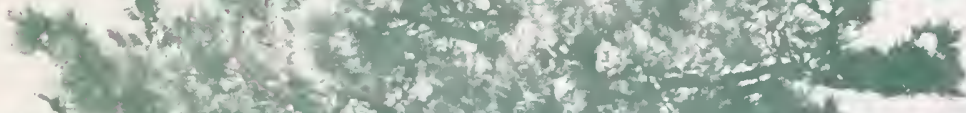

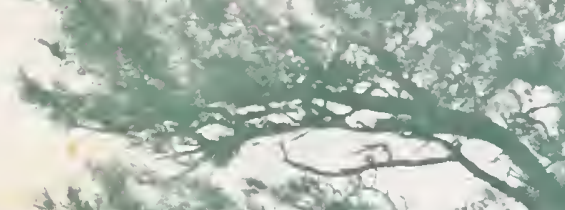

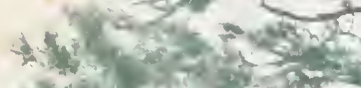

(x)

$+\frac{3}{2}$
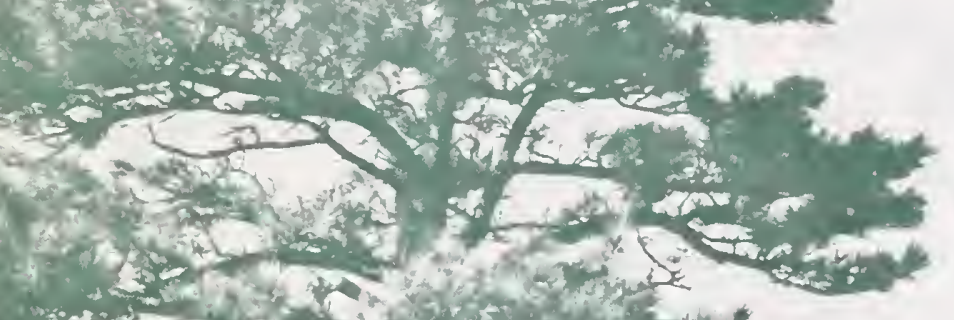

atosinget

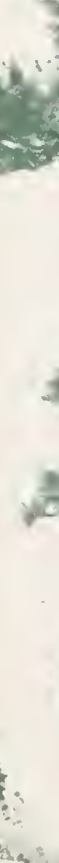

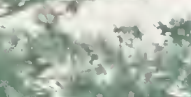
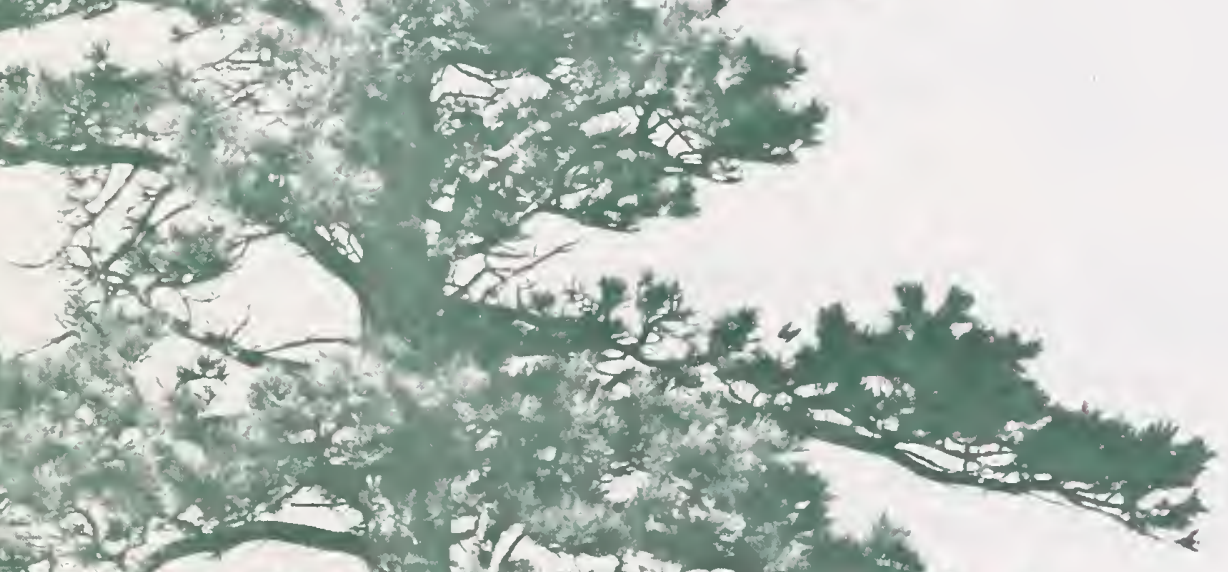

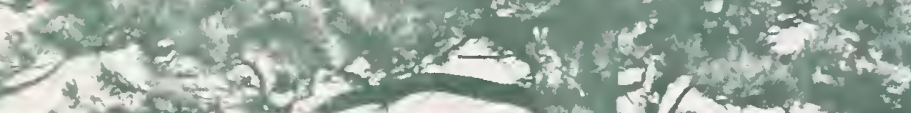
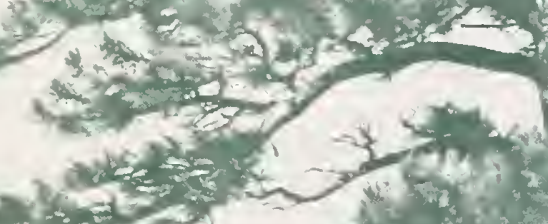

our 5

tort

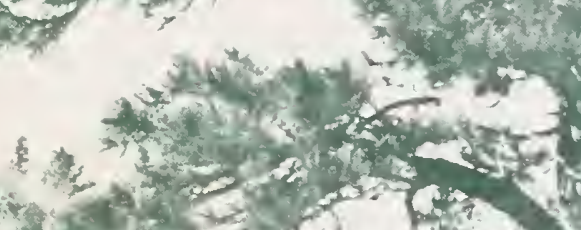

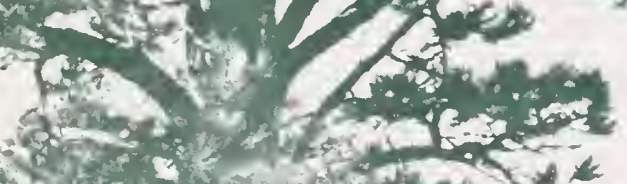

$+2$

(

(
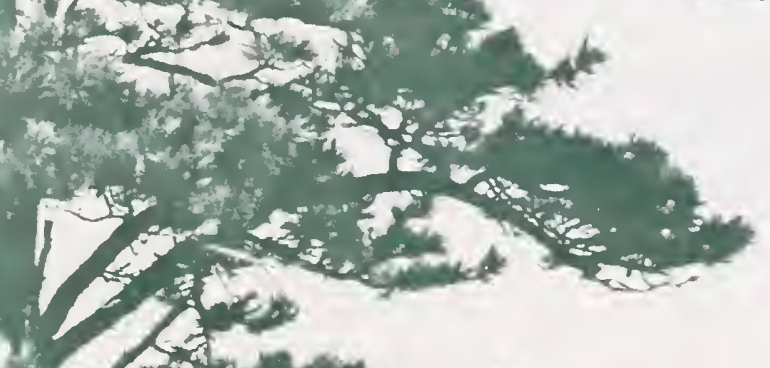

Volume Tables and Point-Sampling Factors for Ponderosa Pine in the Front Range of Colorado
Carleton B. Edminster

Robert T. Beeson

Gary E. Metcalf

Research Paper RM-218

Rocky Mountain Forest and

Range Experiment Station

Forest Service

U.S. Department of Agriculture

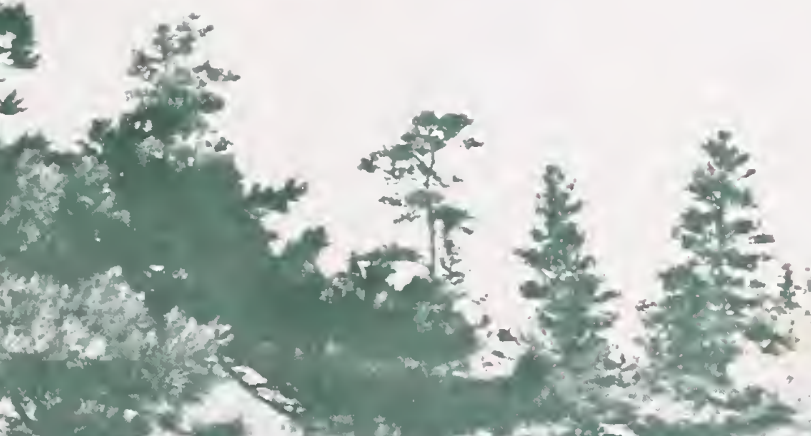

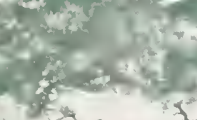

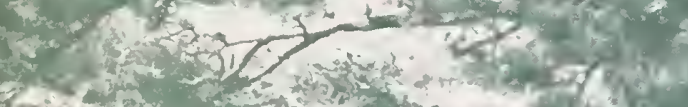

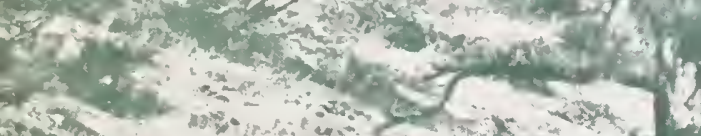
07 . Wh the

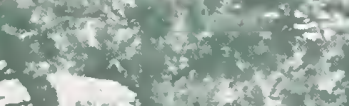

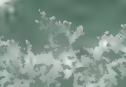

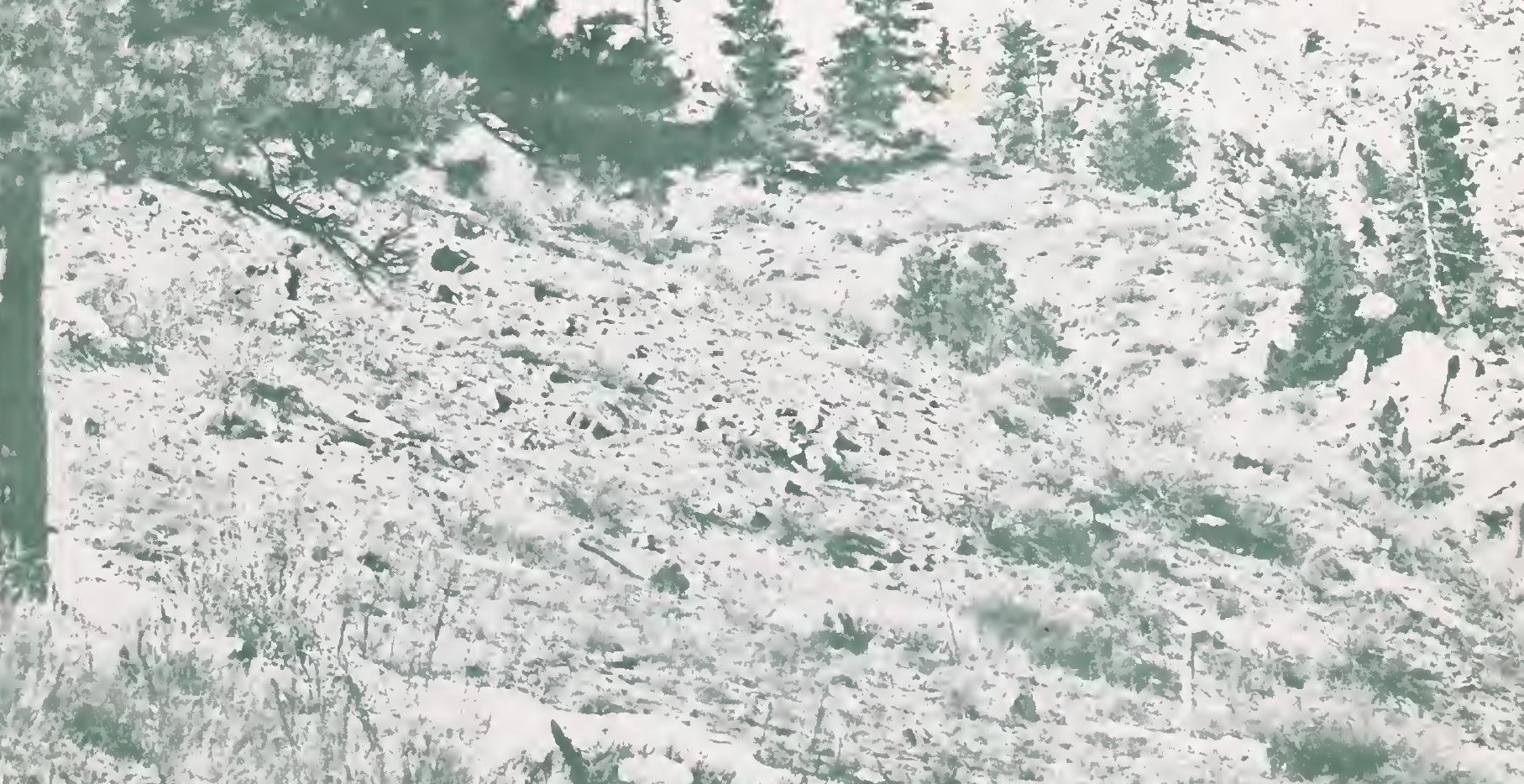

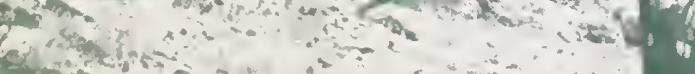

N

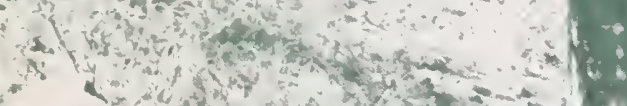

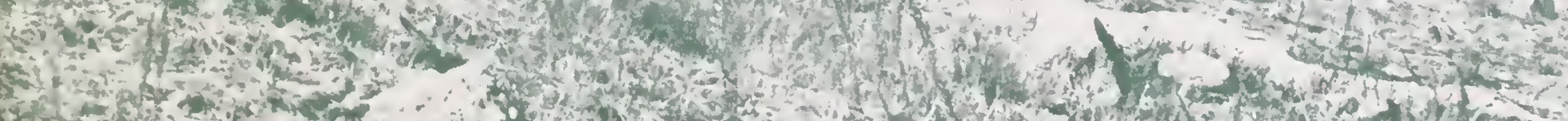


520

Abstract

Volume tables are presented for total cubic feet, merchantable cubic feet to a 4-inch top, board feet Scribner Rule to a 6-inch top, and board feet International 1/4-inck Rule to $\overline{\bar{a}}$ 6-inch top. Pointsampling factor tables are given for $\overline{\bar{m}}$ erchantable volumes per square foot of basal area. Tree heights are expressed as total height in feet and merchantable height in numbers of logs. Volume equations are the form $\underline{V}=\mathrm{a}+\mathrm{bD}_{2}^{2} \mathrm{H}$.

\section{Acknowledgment}

The authors are grateful to personnel of the Arapaho and Roosevelt and Pike and San Isabel National Forests and of the Colorado State Forest Service for measuring sample trees for this study. 


\title{
245 \\ Volume Tables and Point-Sampling Factors for Ponderosa Pine in the Front Range of Colorado.
}

\author{
Carleton B. Edminster, Mensurationist \\ Rocky Mountain Forest and Range Experiment Station' \\ USDA Forest Service \\ Robert T. Beeson, Forest Management Specialist \\ Colorado State Forest Service \\ Gary E. Metcalf, Measurement Specialist \\ Division of Timber Management, Rocky Mountain Region \\ USDA Forest Service
}




\section{Contents}

Page

Management Highlights ..................................... 1

Definitions and Standards ...................................... 1

Explanation of Tables ......................................... 1

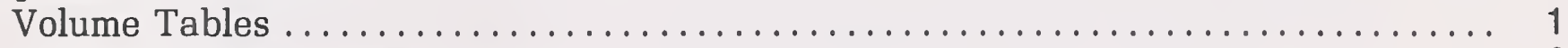

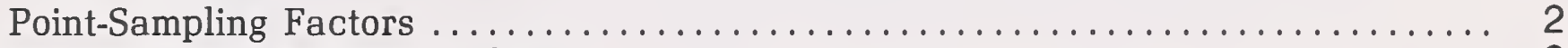

Metric Equations for Cubic Volume .............................. 2

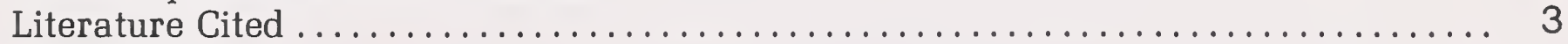

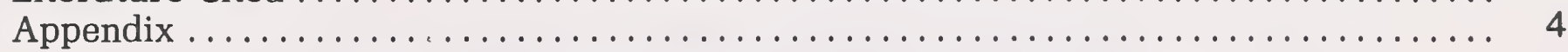

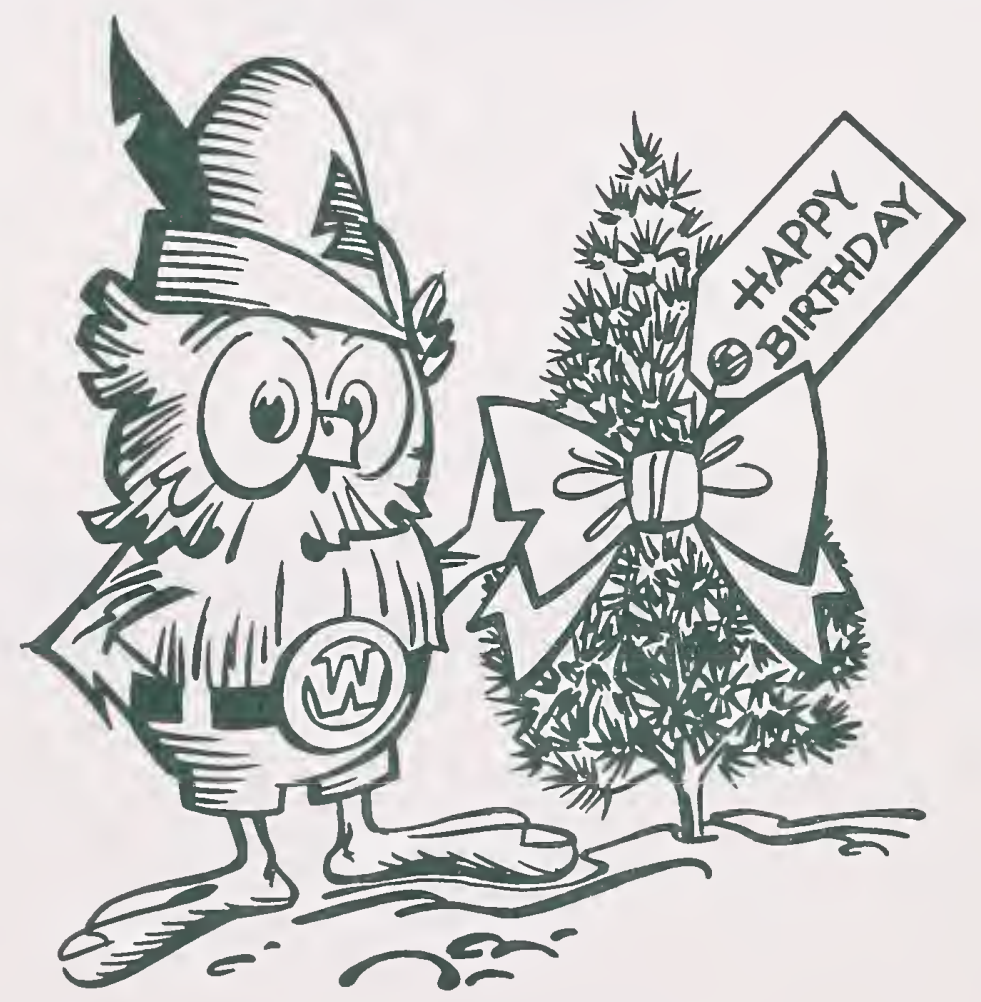

Plant a tree! Mark the 75th birthday of the Forest Service by giving a living gift to future generations. 


\title{
Volume Tables and Point-Sampling Factors for Ponderosa Pine in the Front Range of Colorado
}

\author{
Carleton B. Edminster, Robert T. Beeson, and Gary E. Metcalf
}

\author{
Management Highlights
}

Eleven tables presented here give values and equations needed to determine the volumes of ponderosa pine (Pinus ponderosa var. scopulorum Engelm.) trees in the Front Range of the Rocky Mountains in Colorado. The tables provide:

1. Gross volumes, in cubic feet, of the entire stem.

2. Gross merchantable volumes, in cubic feet, to a 4-inch top.

3. Gross merchantable volumes, in board feet, Scribner and International 1/4-inch Rules, to a 6-inch top.
4. Point-sampling factors giving merchantable volumes in cubic feet and board feet per square foot of basal area.

Stand volumes on an area may be determined from: (1) measurements of all tree diameters and heights, (2) measurements of all tree diameters and sufficient heights to convert the appropriate volume tables to local volume tables (Chapman and Meyer 1949), or (3) tree tallies obtained by point sampling.

\section{Definitions and Standards}

Diameter at breast height (d.b.h.).-Measured to the nearest 0.1 inch, outside the bark, at 4.5 feet above ground level, on the uphill side of the tree. Full-inchdiameter classes, with class midpoints at the $1 / 2$-inch marks, are used in the tables.

Total height.-Measured, in whole feet to the nearest foot, from ground level on the uphill side of the tree upward to the tip. Trees forked below utilization limits described below, stag-topped, or severely deformed were not included in the sample. The midpoints of total height classes in the tables are multiples of 10 feet.

Scaling diameter of logs.-Average diameter inside bark to nearest 0.1 inch, measured at the small end of logs or half-logs.

Minimum top diameters for merchantable volumes. Minimum top diameter inside bark for computation of merchantable cubic-foot volume was 4 inches. For board-foot volume, a minimum top diameter inside bark of 6 inches was used to conform to local practice. Logs with a scaling diameter smaller than 5.6 inches usually were not included in saw-log volume. A few logs with smaller scaling diameters were included to satisfy the "4-foot rule" described below.
Merchantable length in logs.-Measured from 1 foot above ground level on the uphill side of the tree, upward to the limit of saw-log utilization. Each tree was sectioned into as many 16.5 -foot-long logs as possible. An additional half-log, if available, was taken from the uppermost part of the merchantable length. Portions of the bole above the height of minimum top diameter inside bark were included in the uppermost saw-log if the standard $\log$ or half-log length ended within 4 feet above this height. This "4-foot rule" was used to avoid a negative bias in volume determination (Chapman and Meyer 1949).

\section{Explanation of Tables}

General definitions and standards given above apply to all tables listed in the appendix. Explanation of each type of table and suggestions for use follow.

\section{Volume Tables}

Headings and footnotes of each volume table (table 1 and even-numbered tables) give units of volume and height measurement, utilization standards, and volume equations used in compilation. Full-inch-diameter classes and 10-foot-height classes or half-log-length classes were used in all tables. 
The volume tables were developed from linear regressions of $\mathrm{V}$ and $\mathrm{D}^{2} \mathrm{H}$ or $\mathrm{D}^{2} \mathrm{~L}$ of the form:

$$
\mathrm{V}=\mathrm{a}+\mathrm{bD}^{2} \mathrm{H} \text { or } \mathrm{V}=\mathrm{a}+\mathrm{bD}^{2} \mathrm{~L}
$$

where:

$$
\begin{aligned}
& \mathrm{V}=\text { gross volume inside bark in the appropriate } \\
& \text { unit } \\
& \mathrm{D}=\text { d.b.h. outside bark in inches } \\
& \mathrm{H}=\text { total height in feet } \\
& \mathrm{L}=\text { merchantable length in standard logs and half- } \\
& \mathrm{a}, \mathrm{b}=\text { logs }
\end{aligned}
$$

Graphs of $V$ versus $D^{2} \mathrm{H}$ or $\mathrm{D}^{2} \mathrm{~L}$ for all volume relationships did not indicate a nonlinear expression was needed to cover the full range of the basic data. Unfortunately, the linear regression equations for board-foot volumes gave negative estimates for small values of $D^{2} \mathrm{H}$ or $\mathrm{D}^{2} \mathrm{~L}$. To correct this, the volume of a half-log with minimum top diameter has been substituted as described in the footnotes for tables 4, 6, 8, and 10 .

The number of logs in a tree shown in tables 6 and 10 is not necessarily the number that will actually be cut from it. It is the number of logs between the 1 -foot above ground level and the height of minimum top diameter. Volume of nonmerchantable logs below the height of minimum top diameter should be deducted from tree volume by: (1) estimation of scaling diameters and deduction of appropriate log volumes, or (2) use of taper tables to determine scaling diameters and deduction of log volumes. Volume should not be reduced by tallying fewer logs in the tree.

\section{Point-Samping Factors}

Odd-numbered tables from tables 3 through 11 give point-sampling factors for combinations of tree d.b.h. and height or merchantable length. Tabulated volumes per square foot of basal area were obtained from equations given in the table footnotes. These equations were derived by dividing each term of the corresponding tree volume equation by tree basal area in square feet $\left(\mathrm{B}=0.0054542 \mathrm{D}^{2}\right)$.

Point-sample cruising to estimate stand volume can be done in several ways: (1) measure the d.b.h. and height of each tree tallied through the prism, angle gage, or relascope; (2) measure the height of each tallied tree and estimate its d.b.h.; or (3) measure the heights of the tallied trees and make no record of d.b.h.'s. The procedure selected will depend on the precision desired. Relative precision is usually in the order listed above. If the d.b.h. and height of each tallied tree are measured, a volume conversion factor can be selected from the tables or computed from the appropriate equations for each combination of d.b.h. and height. Volume per acre is then computed as follows:
1. Multiply the number of tallied trees in each d.b.h.-height class by the point-sampling factor for the class.

2. Total the products of step 1.

3. Multiply the total of step 2 by the basal area factor of the angle gage used.

4. Divide the product of step 3 by the number of points sampled on the tract.

Considerable time often can be saved if the heights of tallied trees are measured, while d.b.h.'s are estimated and recorded by broad classes. Inspection of the point-sampling factor tables shows that volumes per square foot of basal area, for trees larger than 15 inches d.b.h., often do not differ greatly among trees of a single height class. The increased time spent measuring d.b.h.'s may not increase precision materially. When the distribution of d.b.h.'s and heights inventoried indicates there is little change in volume per square foot within a height class, it is recommended that d.b.h.'s not be recorded at all. Point-sampling factors for each height class can be computed using a procedure similar to deriving a local volume table from a standard table (Chapman and Meyer 1949).

The techniques of point sampling have been described in numerous publications (Dilworth and Bell 1971; Grosenbaugh 1952, 1955, 1958). Procedures for computing tree volumes and point-sampling factors using programmable calculators have been developed by Shepperd (1980).

\section{Metric Equations for Cubic Volume}

The following equations are the metric equivalents (Myers and Edminster 1974) of the cubic-foot volume equations used to develop tables 1-3.

Gross volume of the entire stem in cubic meters:

$$
\mathrm{V}_{\mathrm{m}}=0.0000325 \mathrm{D}_{\mathrm{m}}{ }^{2} \mathrm{H}_{\mathrm{m}}
$$

Gross merchantable volume in cubic meters to a 10-cm top:

$$
\mathrm{V}_{\mathrm{m}}=0.0000311 \mathrm{D}_{\mathrm{m}}{ }^{2} \mathrm{H}_{\mathrm{m}}-0.01265
$$

Gross merchantable volume in cubic meters per square meter of basal area:

$$
\mathrm{V}_{\mathrm{m}} / \mathrm{B}_{\mathrm{m}}=0.39618 \mathrm{H}_{\mathrm{m}}-161.14650 / \mathrm{D}_{\mathrm{m}}^{2}
$$

where:

$\mathrm{V}_{\mathrm{m}}=$ gross volume inside bark in cubic meters

$D_{m}=$ d.b.h. outside bark in centimeters

$\mathrm{H}_{\mathrm{m}}=$ total height in meters

$\mathrm{B}_{\mathrm{m}}=$ tree basal area in square meters 


\section{Literature Cited}

Chapman, Herman H., and Walter H. Meyer. 1949. Forest mensuration. 522 p. McGraw-Hill Book Co., Inc. New York, N.Y.

Dilworth, J. R., and J. F. Bell. 1971. Variable probability sampling-variable plot and three-P. 130 p. Oregon State University Book Stores, Inc., Corvallis.

Grosenbaugh, L. R. 1952. Plotless timber estimatesNew, fast, easy. Journal of Forestry 50:32-37.

Grosenbaugh, L. R. 1955. Better diagnosis and prescription in southern forest management. U.S. Department of Agriculture, Forest Service, Southern Forest Experiment Station, Occasional Paper 145, $27 \mathrm{p}$.
Grosenbaugh, L. R. 1958. Point-sampling and linesampling: Probability theory, geometric implications, synthesis. USDA Forest Service, Southern Forest Experiment Station, Occasional Paper 160, $34 \mathrm{p}$.

Myers, Clifford A., and Carleton B. Edminster. 1974. Conversion of tree-volume equations to the metric system. USDA Forest Service Research Note RM-261, 2 p. Rocky Mountain Forest and Range Experiment Station, Fort Collins, Colo.

Shepperd, Wayne D. 1980. Hand-held-calculator programs for the field forester. USDA Forest Service General Technical Report RM-76. 17 p. Rocky Mountain Forest and Range Experiment Station, Fort Collins, Colo. 


\section{Appendix}

Table 1.-Gross volumes, in cubic feet inside bark, of entire stem including stump and top, ponderosa pine in the Front Range of Colorado

\begin{tabular}{|c|c|c|c|c|c|c|c|c|c|c|c|}
\hline \multirow[b]{2}{*}{ d.b.h. } & \multicolumn{10}{|c|}{ Total height (feet) above ground } & \multirow{2}{*}{$\begin{array}{c}\text { Basis: } \\
\text { trees }\end{array}$} \\
\hline & 10 & 20 & 30 & 40 & 50 & 60 & 70 & 80 & 90 & 100 & \\
\hline \multicolumn{12}{|l|}{ inches } \\
\hline 1 & 0.1 & 0.1 & & & & & & & & & 18 \\
\hline 2 & 0.1 & 0.3 & 0.4 & 0.6 & & & & & & & 21 \\
\hline 3 & 0.3 & 0.6 & 0.8 & 1.1 & & & & & & & 29 \\
\hline 4 & 0.5 & 0.9 & 1.4 & 1.8 & & & & & & & 36 \\
\hline 5 & 0.7 & 1.4 & 2.1 & 2.7 & 3.4 & & & & & & 41 \\
\hline 6 & & 1.9 & 2.9 & 3.8 & 4.8 & & & & & & 36 \\
\hline 7 & & 2.5 & 3.8 & 5.1 & 6.4 & 7.6 & & & & & 40 \\
\hline 8 & & 3.3 & 4.9 & 6.5 & 8.2 & 9.8 & 11.4 & & & & 64 \\
\hline 9 & & 4.1 & 6.1 & 8.2 & 10.2 & 12.2 & 14.3 & & & & 59 \\
\hline 10 & & 5.0 & 7.5 & 10.0 & 12.5 & 14.9 & 17.4 & & & & 77 \\
\hline 11 & & 6.0 & 9.0 & 12.0 & 14.9 & 17.9 & 20.9 & 23.9 & & & 55 \\
\hline 12 & & 7.1 & 10.6 & 14.1 & 17.7 & 21.2 & 24.7 & 28.3 & & & 80 \\
\hline 13 . & & 8.2 & 12.4 & 16.5 & 20.6 & 24.7 & 28.8 & 33.0 & & & 83 \\
\hline 14 & & & 14.3 & 19.0 & 23.8 & 28.5 & 33.3 & 38.0 & & & 68 \\
\hline 15 & & & 16.3 & 21.7 & 27.1 & 32.6 & 38.0 & 43.4 & & & 58 \\
\hline 16 & & & 18.5 & 24.6 & 30.8 & 36.9 & 43.1 & 49.2 & 55.4 & & 52 \\
\hline 17 & & & 20.8 & 27.7 & 34.6 & 41.5 & 48.4 & 55.4 & 62.3 & & 58 \\
\hline 18 & & & 23.2 & 30.9 & 38.7 & 46.4 & 54.1 & 61.9 & 69.6 & & 35 \\
\hline 19 & & & 25.8 & 34.4 & 43.0 & 51.6 & 60.2 & 68.7 & 77.3 & & 18 \\
\hline 20 & & & 28.5 & 38.0 & 47.5 & 57.0 & 66.5 & 76.0 & 85.5 & - & 18 \\
\hline 21 & & & & 41.8 & 52.2 & 62.7 & 73.1 & 83.6 & 94.0 & & 19 \\
\hline 22 & & & & 45.8 & 57.2 & 68.6 & 80.1 & 91.5 & 103.0 & 114.4 & 20 \\
\hline 23 & & & & 49.9 & 62.4 & 74.9 & 87.4 & 99.8 & 112.3 & 124.8 & 8 \\
\hline 24 & & & & & 67.8 & 81.4 & 95.0 & 108.5 & 122.1 & 135.7 & 4 \\
\hline 25 & & & & & 73.5 & 88.2 & 102.9 & 117.6 & 132.3 & 147.0 & 5 \\
\hline 26 & & & & & 79.4 & 95.2 & 111.1 & 127.0 & 142.8 & 158.7 & 3 \\
\hline 27 & & & & & 85.5 & 102.5 & 119.6 & 136.7 & 153.8 & 170.9 & 2 \\
\hline 28 & & & & & & 110.1 & 128.5 & 146.9 & 165.2 & 183.6 & 0 \\
\hline 29 & & & & & & 118.0 & 137.7 & 157.3 & 177.0 & 196.7 & 1 \\
\hline 30 & & & & & & 126.1 & 147.2 & 168.2 & 189.2 & 210.2 & 0 \\
\hline $\begin{array}{l}\text { Basis: } \\
\text { trees }\end{array}$ & 30 & 60 & 157 & 269 & 278 & 166 & 38 & 9 & 1 & 0 & 1,008 \\
\hline
\end{tabular}

Block indicates extent of data.

Computed from: $\mathrm{V}=0.00226 \mathrm{D}^{2} \mathrm{H}$

Standard error of estimate: $\pm 14.16 \%$ of mean; \pm 2.9 cubic feet

Coefficient of determination: 0.9787

Diameter classes full-inch (e.g., 20-inch class includes 20.0 to 20.9 inches d.b.h.) 
Table 2.-Gross merchantable volumes, in cubic feet inside bark, merchantable stem excluding stump and top, ponderosa pine in the Front Range of Colorado. Top diameter 4 inches inside bark. Stump height 1 foot

\begin{tabular}{|c|c|c|c|c|c|c|c|c|c|c|}
\hline \multirow[b]{2}{*}{ d.b.h. } & \multicolumn{9}{|c|}{ Total height (feet) above ground } & \multirow{2}{*}{$\begin{array}{l}\text { Basis: } \\
\text { trees }\end{array}$} \\
\hline & 20 & 30 & 40 & 50 & 60 & 70 & 80 & 90 & 100 & \\
\hline \multicolumn{11}{|l|}{ inches } \\
\hline 5 & 0.9 & 1.5 & 2.2 & 2.8 & & & & & & 41 \\
\hline 6 & 1.4 & 2.3 & 3.2 & 4.1 & & & & & & 36 \\
\hline 7 & 2.0 & 3.2 & 4.4 & 5.6 & 6.8 & & & & & 40 \\
\hline 8 & 2.7 & 4.2 & 5.8 & 7.4 & 8.9 & 10.5 & & & & 64 \\
\hline 9 & 3.5 & 5.4 & 7.4 & 9.3 & 11.2 & 13.2 & & & & 59 \\
\hline 10 & 4.3 & 6.7 & 9.1 & 11.5 & 13.8 & 16.2 & & & & 77 \\
\hline 11 & 5.3 & 8.1 & 11.0 & 13.8 & 16.7 & 19.5 & 22.4 & & & 55 \\
\hline 12 & 6.3 & 9.7 & 13.1 & 16.4 & 19.8 & 23.2 & 26.6 & & & 80 \\
\hline 13 & 7.4 & 11.4 & 15.3 & 19.2 & 23.2 & 27.1 & 31.0 & & & 83 \\
\hline 14 & & 13.2 & 17.7 & 22.3 & 26.8 & 31.3 & 35.9 & & & 68 \\
\hline 15 & & 15.1 & 20.3 & 25.5 & 30.7 & 35.9 & 41.1 & & & 58 \\
\hline 16 & & 17.2 & 23.1 & 29.0 & 34.8 & 40.7 & 46.6 & 52.5 & & 52 \\
\hline 17 & & 19.4 & 26.0 & 32.6 & 39.2 & 45.9 & 52.5 & 59.1 & & 58 \\
\hline 18 & & 21.7 & 29.1 & 36.5 & 43.9 & 51.3 & 58.7 & 66.1 & & 35 \\
\hline 19 & & 24.2 & 32.4 & 40.6 & 48.8 & 57.0 & 65.3 & 73.5 & & 18 \\
\hline 20 & & 26.8 & 35.9 & 44.9 & 54.0 & 63.1 & 72.2 & 81.2 & & 18 \\
\hline 21 & & & 39.5 & 49.5 & 59.5 & 69.4 & 79.4 & 89.4 & & 19 \\
\hline 22 & & & 43.3 & 54.2 & 65.2 & 76.1 & 87.0 & 98.0 & 108.9 & 20 \\
\hline 23 & & & 47.3 & 59.2 & 71.1 & 83.1 & 95.0 & 106.9 & 118.8 & 8 \\
\hline 24 & & & & 64.4 & 77.3 & 90.3 & 103.3 & 116.2 & 129.2 & 4 \\
\hline 25 & & & & 69.8 & 83.8 & 97.9 & 111.9 & 126.0 & 140.0 & 5 \\
\hline 26 & & & & 75.4 & 90.6 & 105.7 & 120.9 & 136.1 & 151.2 & 3 \\
\hline 27 & & & & 81.2 & 97.6 & 113.9 & 130.2 & 146.6 & 162.9 & 2 \\
\hline 28 & & & & & 104.8 & 122.4 & 139.9 & 157.5 & 175.0 & 0 \\
\hline 29 & & & & & 112.3 & 131.1 & 149.9 & 168.7 & 187.5 & 1 \\
\hline 30 & & & & & 120.1 & 140.2 & 160.3 & 180.4 & 200.5 & 0 \\
\hline $\begin{array}{l}\text { Basis: } \\
\text { trees }\end{array}$ & 11 & 132 & 269 & 278 & 166 & 38 & 9 & 1 & 0 & 904 \\
\hline
\end{tabular}

Block indicates extent of data.

Computed from: $\mathrm{V}=0.00216 \mathrm{D}^{2} \mathrm{H}-0.44670$

Standard error of estimate: $\pm 14.29 \%$ of mean; \pm 3.0 cubic feet

Coefficient of determination: 0.9744

Diameter classes full-inch (e.g., 20-inch class includes 20.0 to 20.9 inches d.b.h.) 
Table 3.- Gross merchantable volumes, in cubic feet inside bark per square foot of basal area, merchantable stem excluding stump and top, ponderosa pine in the Front Range of Colorado. Top diameter 4 inches inside bark. Stump height 1 foot

\begin{tabular}{|c|c|c|c|c|c|c|c|c|c|}
\hline \multirow[b]{2}{*}{ d.b.h. } & \multicolumn{9}{|c|}{ Total height (feet) above ground } \\
\hline & 20 & 30 & 40 & 50 & 60 & 70 & 80 & 90 & 100 \\
\hline \multicolumn{10}{|l|}{ inches } \\
\hline 5 & 5.2 & 9.2 & 13.1 & 17.1 & & & & & \\
\hline 6 & 6.0 & 9.9 & 13.9 & 17.9 & & & & & \\
\hline 7 & 6.5 & 10.4 & 14.4 & 18.3 & 22.3 & & & & \\
\hline 8 & 6.8 & 10.7 & 14.7 & 18.7 & 22.6 & 26.6 & & & \\
\hline 9 & 7.0 & 11.0 & 14.9 & 18.9 & 22.9 & 26.8 & & & \\
\hline 10 & 7.2 & 11.1 & 15.1 & 19.1 & 23.0 & 27.0 & & & \\
\hline 11 & 7.3 & 11.3 & 15.2 & 19.2 & 23.1 & 27.1 & 31.1 & & \\
\hline 12 & 7.4 & 11.4 & 15.3 & 19.3 & 23.2 & 27.2 & 31.2 & & \\
\hline 13 & 7.5 & 11.4 & 15.4 & 19.4 & 23.3 & 27.3 & 31.2 & & \\
\hline 14 & & 11.5 & 15.5 & 19.4 & 23.4 & 27.3 & 31.3 & & \\
\hline 15 & & 11.5 & 15.5 & 19.5 & 23.4 & 27.4 & 31.3 & & \\
\hline 16 & & 11.6 & 15.5 & 19.5 & 23.5 & 27.4 & 31.4 & 35.3 & \\
\hline 17 & & 11.6 & 15.6 & 19.5 & 23.5 & 27.5 & 31.4 & 35.4 & . \\
\hline 18 & & 11.6 & 15.6 & 19.6 & 23.5 & 27.5 & 31.4 & 35.4 & \\
\hline 19 & & 11.7 & 15.6 & 19.6 & 23.5 & 27.5 & 31.5 & 35.4 & \\
\hline 20 & & 11.7 & 15.6 & 19.6 & 23.6 & 27.5 & 31.5 & 35.4 & \\
\hline 21 & & & 15.7 & 19.6 & 23.6 & 27.5 & 31.5 & 35.5 & \\
\hline 22 & & & 15.7 & 19.6 & 23.6 & 27.6 & 31.5 & 35.5 & 39.4 \\
\hline 23 & & & 15.7 & 19.7 & 23.6 & 27.6 & 31.5 & 35.5 & 39.5 \\
\hline 24 & & & & 19.7 & 23.6 & 27.6 & 31.5 & 35.5 & 39.5 \\
\hline 25 & & & & 19.7 & 23.6 & 27.6 & 31.6 & 35.5 & 39.5 \\
\hline 26 & & & & 19.7 & 23.6 & 27.6 & 31.6 & 35.5 & 39.5 \\
\hline 27 & & & & 19.7 & 23.7 & 27.6 & 31.6 & 35.5 & 39.5 \\
\hline 28 & & & & & 23.7 & 27.6 & 31.6 & 35.5 & 39.5 \\
\hline 29 & & & & & 23.7 & 27.6 & 31.6 & 35.5 & 39.5 \\
\hline 30 & & & & & 23.7 & 27.6 & 31.6 & 35.6 & 39.5 \\
\hline
\end{tabular}

Computed from: $V / B=0.39603 \mathrm{H}-81.90019 / D^{2}$

Diameter classes full-inch (e.g., 20-inch class includes 20.0 to 20.9 inches d.b.h.) 
Table 4.-Gross volumes, in board feet inside bark Scribner Rule, merchantable stem excluding stump and top, ponderosa pine in the Front Range of Colorado. Top diameter 6 inches inside bark. Stump height 1 foot

\begin{tabular}{|c|c|c|c|c|c|c|c|c|c|}
\hline \multirow[b]{2}{*}{ d.b.h. } & \multicolumn{8}{|c|}{ Total height (feet) above ground } & \multirow{2}{*}{$\begin{array}{l}\text { Basis: } \\
\text { trees }\end{array}$} \\
\hline & 30 & 40 & 50 & 60 & 70 & 80 & 90 & 100 & \\
\hline \multicolumn{10}{|l|}{ inches } \\
\hline 7 & 8 & 8 & 8 & 14 & & & & & 26 \\
\hline 8 & 8 & 9 & 17 & 25 & 34 & & & & 63 \\
\hline 9 & 8 & 17 & 27 & 38 & 48 & & & & 58 \\
\hline 10 & 13 & 26 & 39 & 51 & 64 & & & & 77 \\
\hline 11 & 21 & 36 & 51 & 67 & 82 & 97 & & & 55 \\
\hline 12 & 29 & 47 & 65 & 83 & 101 & 119 & & & 77 \\
\hline 13 & 38 & 59 & 80 & 101 & 122 & 143 & & & 83 \\
\hline 14 & 48 & 72 & 96 & 120 & 145 & 169 & & & 68 \\
\hline 15 & 58 & 86 & 113 & 141 & 169 & 196 & & & 58 \\
\hline 16 & 69 & 101 & 132 & 163 & 194 & 226 & 257 & & 51 \\
\hline 17 & 81 & 116 & 151 & 187 & 222 & 257 & 292 & & 58 \\
\hline 18 & 93 & 133 & 172 & 211 & 251 & 290 & 329 & & 35 \\
\hline 19 & 107 & 150 & 194 & 238 & 281 & 325 & 369 & & 18 \\
\hline 20 & 120 & 169 & 217 & 265 & 313 & 362 & 410 & & 18 \\
\hline 21 & & 188 & 241 & 294 & 347 & 400 & 453 & & 19 \\
\hline 22 & & 208 & 266 & 324 & 383 & 441 & 499 & 557 & 20 \\
\hline 23 & & 229 & 293 & 356 & 420 & 483 & 547 & 610 & 8 \\
\hline 24 & & & 320 & 389 & 458 & 527 & 596 & 665 & 4 \\
\hline 25 & & & 349 & 424 & 498 & 573 & 648 & 723 & 5 \\
\hline 26 & & & 379 & 460 & 540 & 621 & 702 & 782 & 3 \\
\hline 27 & & & 410 & 497 & 584 & 671 & 757 & 844 & 2 \\
\hline 28 & & & & 535 & 629 & 722 & 815 & 909 & 0 \\
\hline 29 & & & & 575 & 675 & 775 & 875 & 975 & 1 \\
\hline 30 & & & & 617 & 724 & 831 & 937 & 1,044 & 0 \\
\hline Basis: & & & & & & & & & \\
\hline trees & 70 & 247 & 276 & 166 & 38 & 9 & 1 & 0 & 807 \\
\hline
\end{tabular}

Computed from: $V=8$ for $D^{2} H$ to 2,$830 ; V=0.01149 D^{2} H-24.5404$ for $D^{2} H$ larger than 2,830 Standard error of estimate: $\pm 25.36 \%$ of mean; \pm 26 board feet

Coefficient of determination: 0.9351

Diameter classes full-inch (e.g., 20-inch class includes 20.0 to 20.9 inches d.b.h.) 
Table 5.-Gross volumes, in board feet inside bark Scribner Rule per square foot of basal area, merchantable stem excluding stump and top, ponderosa pine in the Front Range of Colorado. Top diameter 6 inches inside-bark. Stump height 1 foot

\begin{tabular}{|c|c|c|c|c|c|c|c|c|}
\hline \multirow[b]{2}{*}{ d.b.h. } & \multicolumn{8}{|c|}{ Total height (feet) above ground } \\
\hline & 30 & 40 & 50 & 60 & 70 & 80 & 90 & 100 \\
\hline \multicolumn{9}{|l|}{ inches } \\
\hline 7 & 26 & 26 & 26 & 46 & & & & \\
\hline 8 & 20 & 22 & 43 & 64 & 85 & & & \\
\hline 9 & 16 & 34 & 55 & 77 & 98 & & & \\
\hline 10 & 22 & 43 & 65 & 86 & 107 & & & \\
\hline 11 & 29 & 50 & 71 & 92 & 113 & 135 & & \\
\hline 12 & 34 & 55 & 77 & 98 & 119 & 140 & & \\
\hline 13 & 39 & 60 & 81 & 102 & 123 & 144 & & \\
\hline 14 & 42 & 63 & 84 & 105 & 126 & 147 & & \\
\hline 15 & 44 & 66 & 87 & 108 & 129 & 150 & & \\
\hline 16 & 47 & 68 & 89 & 110 & 131 & 152 & 173 & \\
\hline 17 & 49 & 70 & 91 & 112 & 133 & 154 & 175 & \\
\hline 18 & 50 & 71 & 92 & 113 & 134 & 155 & 176 & \\
\hline 19 & 51 & 72 & 93 & 115 & 136 & 157 & 178 & \\
\hline 20 & 52 & 74 & 95 & 116 & 137 & 158 & 179 & \\
\hline 21 & & 75 & 96 & 117 & 138 & 159 & 180 & \\
\hline 22 & & 75 & 96 & 118 & 139 & 160 & 181 & 202 \\
\hline 23 & & 76 & 97 & 118 & 139 & 160 & 181 & 203 \\
\hline 24 & & & 98 & 119 & 140 & 161 & 182 & 203 \\
\hline 25 & & & 98 & 119 & 141 & 162 & 183 & 204 \\
\hline 26 & & & 99 & 120 & 141 & 162 & 183 & 204 \\
\hline 27 & & & 99 & 120 & 142 & 163 & 184 & 205 \\
\hline 28 & & & & 121 & 142 & 163 & 184 & 205 \\
\hline 29 & & & & 121 & 142 & 163 & 184 & 205 \\
\hline 30 & & & & 122 & 143 & 164 & 185 & 206 \\
\hline
\end{tabular}


Table 6.-Gross volumes, in board feet inside Scribner Rule, merchantable stem excluding stump and top, ponderosa pine in the Front Range of Colorado. Top diameter 6 inches inside bark. Stump height 1 foot

\begin{tabular}{|c|c|c|c|c|c|c|c|c|c|c|c|}
\hline \multirow[b]{2}{*}{ d.b.h. } & \multicolumn{10}{|c|}{ Number of 16 -foot logs to 6 -inch top } & \multirow{2}{*}{$\begin{array}{l}\text { Basis: } \\
\text { trees }\end{array}$} \\
\hline & 0.5 & 1.0 & 1.5 & 2.0 & 2.5 & 3.0 & 3.5 & 4.0 & 4.5 & 5.0 & \\
\hline \multicolumn{12}{|l|}{ inches } \\
\hline 7 & 8 & 8 & 13 & 19 & & & & & & & 26 \\
\hline 8 & 8 & 10 & 18 & 26 & 35 & & & & & & 63 \\
\hline 9 & 8 & 14 & 24 & 34 & 45 & & & & & & 58 \\
\hline 10 & 8 & 19 & 31 & 44 & 56 & & & & & & 77 \\
\hline 11 & 9 & 24 & 39 & 53 & 68 & 83 & & & & & 55 \\
\hline 12 & 11 & 29 & 47 & 64 & 82 & 100 & 117 & & & & 77 \\
\hline 13 & 14 & 35 & 55 & 76 & 97 & 117 & 138 & 158 & & & 83 \\
\hline 14 & & 41 & 65 & 89 & 112 & 136 & 160 & 183 & & & 68 \\
\hline 15 & & 48 & 75 & 102 & 129 & 156 & 183 & 211 & & & 58 \\
\hline 16 & & 55 & 86 & 117 & 147 & 178 & 209 & 239 & 270 & & 51 \\
\hline 17 & & 63 & 97 & 132 & 166 & 201 & 236 & 270 & 305 & & 58 \\
\hline 18 & & 71 & 110 & 148 & 187 & 225 & 264 & 303 & 341 & & 35 \\
\hline 19 & & 80 & 122 & 165 & 208 & 251 & 294 & 337 & 380 & & 18 \\
\hline 20 & & 89 & 136 & 183 & 231 & 278 & 326 & 373 & 420 & & 18 \\
\hline 21 & & & 150 & 202 & 254 & 307 & 359 & 411 & 463 & & 19 \\
\hline 22 & & & 165 & 222 & 279 & 336 & 393 & 451 & 508 & 565 & 20 \\
\hline 23 & & & 181 & 243 & 305 & 367 & 430 & 492 & 554 & 617 & 8 \\
\hline 24 & & & & 265 & 332 & 400 & 468 & 535 & 603 & 671 & 4 \\
\hline 25 & & & & & 360 & 434 & 507 & 580 & 654 & 727 & 5 \\
\hline 26 & & & & & 390 & 469 & 548 & 627 & 707 & 786 & 3 \\
\hline 27 & & & & & 420 & 506 & 591 & 676 & 761 & 847 & 2 \\
\hline 28 & & & & & 452 & 543 & 635 & 727 & 818 & 910 & 0 \\
\hline 29 & & & & & 485 & 583 & 681 & 779 & 877 & 975 & 1 \\
\hline 30 & & & & & & 623 & 728 & 833 & 938 & 1,043 & 0 \\
\hline $\begin{array}{l}\text { Basis: } \\
\text { trees }\end{array}$ & 44 & 104 & 187 & 212 & & & 18 & & & , & \\
\hline & 44 & 104 & 181 & 212 & 150 & 81 & 18 & 4 & 1 & 0 & 807 \\
\hline
\end{tabular}

Block indicates extent of data.

Computed from: $V=8$ for $D^{2} L$ to $63 ; V=0.22556 D^{2} L-6.22508$ for $D^{2} L$ larger than 63

Standard error of estimate: $\pm 23.15 \%$ of mean; \pm 24 board feet

Coefficient of determination: 0.9459

Diameter classes full-inch (e.g., 20-inch class includes 20.0 to 20.9 inches d.b.h.) 
Table 7.- Gross volumes, in board feet inside bark Scribner Rule per square foot of basal area, merchantable stem excluding stump and top, ponderosa pine in the Front Range of Colorado. Top diameter 6 inches inside bark. Stump height 1 foot

\begin{tabular}{|c|c|c|c|c|c|c|c|c|c|c|}
\hline \multirow[b]{2}{*}{ d.b.h. } & \multicolumn{10}{|c|}{ Number of 16 -foot logs to 6 -inch top } \\
\hline & 0.5 & 1.0 & 1.5 & 2.0 & 2.5 & 3.0 & 3.5 & 4.0 & 4.5 & 5.0 \\
\hline \multicolumn{11}{|l|}{ inches } \\
\hline 7 & 26 & 26 & 42 & 62 & & & & & & \\
\hline 8 & 20 & 26 & 46 & 67 & 88 & & & & & \\
\hline 9 & 16 & 29 & 49 & 70 & 91 & & & & & \\
\hline 10 & 13 & 31 & 52 & 72 & 93 & & & & & \\
\hline 11 & 12 & 33 & 53 & 74 & 95 & 115 & & & & \\
\hline 12 & 13 & 34 & 55 & 75 & 96 & 117 & 137 & & & \\
\hline 13 & 14 & 35 & 56 & 76 & 97 & 118 & 138 & 159 & & \\
\hline 14 & & 36 & 57 & 77 & 98 & 119 & 139 & 160 & & \\
\hline 15 & & 37 & 57 & 78 & 99 & 119 & 140 & 161 & & \\
\hline 16 & & 37 & 58 & 79 & 99 & 120 & 141 & 161 & 182 & \\
\hline 17 & & 38 & 58 & 79 & 100 & 120 & 141 & 162 & 182 & \\
\hline 18 & & 38 & 59 & 79 & 100 & 121 & 141 & 162 & 183 & \\
\hline 19 & & 38 & 59 & 80 & 100 & 121 & 142 & 162 & 183 & \\
\hline 20 & & 39 & 59 & 80 & 101 & 121 & 142 & 163 & 183 & \\
\hline 21 & & & 60 & 80 & 101 & 122 & 142 & 163 & 184 & \\
\hline 22 & & & 60 & 80 & 101 & 122 & 142 & 163 & 184 & 205 \\
\hline 23 & & & 60 & 81 & 101 & 122 & 143 & 163 & 184 & 205 \\
\hline 24 & & & & 81 & 101 & 122 & 143 & 164 & 184 & 205 \\
\hline 25 & & & & & 102 & 122 & 143 & 164 & 184 & 205 \\
\hline 26 & & & & & 102 & 122 & 143 & 164 & 184 & 205 \\
\hline 27 & & & & & 102 & 123 & 143 & 164 & 185 & 205 \\
\hline 28 & & & & & 102 & 123 & 143 & 164 & 185 & 205 \\
\hline 29 & & & & & 102 & 123 & 143 & 164 & 185 & 205 \\
\hline 30 & & & & & & 123 & 144 & 164 & 185 & 206 \\
\hline
\end{tabular}

Computed from: $V / B=1,466.75956 / D^{2}$ for $D^{2} L$ to $63 ; V / B=41.35529 L-1,141.33695 / D^{2}$ for $D^{2} L$ larger than 63 Diameter classes full-inch (e.g., 20-inch class includes 20.0 to 20.9 inches d.b.h.) 
Table 8.-Gross volumes, in board feet inside bark International 1/4-inch Rule, merchantable stem excluding stump and top, ponderosa pine in the Front Range of Colorado. Top diameter 6 inches inside bark. Stump height 1 foot

\begin{tabular}{|c|c|c|c|c|c|c|c|c|c|}
\hline \multirow[b]{2}{*}{ d.b.h. } & \multicolumn{8}{|c|}{ Total height (feet) above ground } & \multirow{2}{*}{$\begin{array}{l}\text { Basis: } \\
\text { trees }\end{array}$} \\
\hline & 30 & 40 & 50 & 60 & 70 & 80 & 90 & 100 & \\
\hline \multicolumn{10}{|l|}{ inches } \\
\hline 7 & 9 & 9 & 13 & 20 & & & & & 26 \\
\hline 8 & 9 & 14 & 23 & 32 & 41 & & & & 63 \\
\hline 9 & 11 & 23 & 34 & 46 & 58 & & & & 58 \\
\hline 10 & 19 & 33 & 47 & 61 & 76 & & & & 77 \\
\hline 11 & 27 & 44 & 61 & 78 & 95 & 112 & & & 55 \\
\hline 12 & 37 & 57 & 77 & 97 & 117 & 137 & & & 77 \\
\hline 13 & 47 & 70 & 94 & 117 & 140 & 164 & & & 83 \\
\hline 14 & 58 & 85 & 112 & 139 & 166 & 193 & & & 68 \\
\hline 15 & 69 & 100 & 131 & 162 & 193 & 224 & & & 58 \\
\hline 16 & 81 & 116 & 151 & 186 & 221 & 256 & 292 & & 51 \\
\hline 17 & 95 & 134 & 173 & 213 & 252 & 291 & 331 & & 58 \\
\hline 18 & 108 & 152 & 196 & 240 & 285 & 329 & 373 & & 35 \\
\hline 19 & 123 & 172 & 221 & 270 & 319 & 368 & 417 & & 18 \\
\hline 20 & 139 & 193 & 247 & 301 & 355 & 409 & 463 & & 18 \\
\hline 21 & & 214 & 274 & 333 & 393 & 452 & 511 & & 19 \\
\hline 22 & & 237 & 302 & 367 & 432 & 497 & 562 & 627 & 20 \\
\hline 23 & & 260 & 332 & 403 & 474 & 545 & 616 & 687 & 8 \\
\hline 24 & & & 362 & 440 & 517 & 594 & 671 & 748 & 4 \\
\hline 25 & & & 395 & 478 & 562 & 645 & 729 & 813 & 5 \\
\hline 26 & & & 428 & 518 & 609 & 699 & 789 & 880 & 3 \\
\hline 27 & & & 463 & 560 & 657 & 754 & 852 & 949 & 2 \\
\hline 28 & & & & 603 & 708 & 812 & 917 & 1,021 & 0 \\
\hline 29 & & & & 648 & 760 & 872 & 984 & 1,096 & 1 \\
\hline 30 & & & & 694 & 814 & 933 & 1,053 & 1,173 & 0 \\
\hline Basis: & & & & & & & & & \\
\hline trees & 70 & 247 & 216 & 166 & 38 & 9 & 1 & 0 & 807 \\
\hline
\end{tabular}

Block indicates extent of data

Computed from: $V=9$ for $D^{2} H$ to 2,$535 ; V=0.01286 D^{2} H-23.5932$ for $D^{2} H$ larger than 2,535

Standard error of estimate: $\pm 23.36 \%$ of mean; \pm 28 board feet

Coefficient of determination: 0.9408

Diameter classes full-inch (e.g., 20-inch class includes 20.0 to 20.9 inches d.b.h.) 
Table 9.-Gross volumes, in board feet inside bark International 1/4-inch Rule per square foot of basal area, merchantable stem excluding stump and top, ponderosa pine in the Front Range of Colorado. Top diameter 6 inches inside bark. Stump height 1 foot

\begin{tabular}{|c|c|c|c|c|c|c|c|c|}
\hline \multirow[b]{2}{*}{ d.b.h. } & \multicolumn{8}{|c|}{ Total height (feet) above ground } \\
\hline & 30 & 40 & 50 & 60 & 70 & 80 & 90 & 100 \\
\hline \multicolumn{9}{|l|}{ inches } \\
\hline 7 & 29 & 29 & 41 & 65 & & & & \\
\hline 8 & 23 & 34 & 58 & 82 & 105 & & & \\
\hline 9 & 23 & 46 & 70 & 94 & 117 & & & \\
\hline 10 & 31 & 55 & 79 & 102 & 126 & & & \\
\hline 11 & 38 & 62 & 85 & 109 & 132 & 156 & & \\
\hline 12 & 43 & 67 & 90 & 114 & 137 & 161 & & \\
\hline 13 & 47 & 71 & 94 & 118 & 141 & 165 & & \\
\hline 14 & 50 & 74 & 97 & 121 & 144 & 168 & & \\
\hline 15 & 53 & 76 & 100 & 123 & 147 & 171 & & \\
\hline 16 & 55 & 78 & 102 & 126 & 149 & 173 & 196 & \\
\hline 17 & 57 & 80 & 104 & 127 & 151 & 175 & 198 & \\
\hline 18 & 58 & 82 & 105 & 129 & 152 & 176 & 200 & \\
\hline 19 & 59 & 83 & 107 & 130 & 154 & 177 & 201 & \\
\hline 20 & 60 & 84 & 108 & 131 & 155 & 178 & 202 & \\
\hline 21 & & 85 & 109 & 132 & 156 & 179 & 203 & \\
\hline 22 & & 86 & 109 & 133 & 157 & 180 & 204 & 227 \\
\hline 23 & & 86 & 110 & 134 & 157 & 181 & 204 & 228 \\
\hline 24 & & & 111 & 134 & 158 & 181 & 205 & 229 \\
\hline 25 & & & 111 & 135 & 158 & 182 & 206 & 229 \\
\hline 26 & & & 112 & 135 & 159 & 182 & 206 & 230 \\
\hline 27 & & & 112 & 136 & 159 & 183 & 206 & 230 \\
\hline 28 & & & & 136 & 160 & 183 & 207 & 230 \\
\hline 29 & & & & 136 & 160 & 184 & 207 & 231 \\
\hline 30 & & & & 137 & 160 & 184 & 208 & 231 \\
\hline
\end{tabular}


Table 10.-Gross volumes, in board feet inside bark International 1/4-inch Rule, merchantable stem excluding stump and top, ponderosa pine in the Front Range of Colorado. Top diameter 6 inches inside bark. Stump height 1 foot

\begin{tabular}{|c|c|c|c|c|c|c|c|c|c|c|c|}
\hline \multirow[b]{2}{*}{ d.b.h. } & \multicolumn{10}{|c|}{ Number of $16 \cdot$ foot logs to 6 -inch top } & \multirow{2}{*}{$\begin{array}{l}\text { Basis: } \\
\text { trees }\end{array}$} \\
\hline & 0.5 & 1.0 & 1.5 & 2.0 & 2.5 & 3.0 & 3.5 & 4.0 & 4.5 & 5.0 & \\
\hline \multicolumn{12}{|l|}{ inches } \\
\hline 7 & 9 & 11 & 18 & 25 & & & & & & & 26 \\
\hline 8 & 9 & 15 & 24 & 33 & 43 & & & & & & 63 \\
\hline 9 & 9 & 20 & 31 & 43 & 54 & & & & & & 58 \\
\hline 10 & 11 & 25 & 39 & 53 & 67 & & & & & & 77 \\
\hline 11 & 14 & 30 & 47 & 64 & 80 & 97 & & & & & 55 \\
\hline 12 & 17 & 36 & 56 & 76 & 96 & 115 & 135 & & & & 77 \\
\hline 13 & 20 & 43 & 66 & 89 & 112 & 135 & 158 & 181 & & & 83 \\
\hline 14 & & 50 & 77 & 103 & 130 & 156 & 183 & 209 & & & 68 \\
\hline 15 & & 58 & 88 & 118 & 149 & 179 & 209 & 240 & & & 58 \\
\hline 16 & & 66 & 100 & 134 & 169 & 203 & 238 & 272 & 306 & & 51 \\
\hline 17 & & 74 & 113 & 152 & 190 & 229 & 268 & 306 & 345 & & 58 \\
\hline 18 & & 83 & 127 & 170 & 213 & 256 & 299 & 343 & 386 & & 35 \\
\hline 19 & & 93 & 141 & 189 & 237 & 285 & 333 & 381 & 429 & & 18 \\
\hline 20 & & 103 & 156 & 209 & 262 & 315 & 368 & 421 & 474 & & 18 \\
\hline 21 & & & 172 & 230 & 289 & 347 & 405 & 464 & 522 & & 19 \\
\hline 22 & & & 189 & 253 & 316 & 380 & 444 & 508 & 572 & 636 & 20 \\
\hline 23 & & & 206 & 276 & 346 & 415 & 485 & 555 & 624 & 694 & 8 \\
\hline 24 & & & & 300 & 376 & 452 & 527 & 603 & 679 & 755 & 4 \\
\hline 25 & & & & & 407 & 489 & 572 & 654 & 736 & 818 & 5 \\
\hline 26 & & & & & 440 & 529 & 618 & 706 & 795 & 883 & 3 \\
\hline 27 & & & & & 474 & 570 & 665 & 761 & 856 & 952 & 2 \\
\hline 28 & & & & & 510 & 612 & 715 & 817 & 920 & 1,022 & 0 \\
\hline 29 & & & & & 546 & 656 & 766 & 876 & 986 & 1,096 & 1 \\
\hline 30 & & & & & & 702 & 819 & 936 & 1,054 & 1,171 & 0 \\
\hline Basis: & & & & & & & & & & & \\
\hline trees & 44 & 104 & 187 & 212 & 150 & 87 & 18 & 4 & 1 & 0 & 807 \\
\hline
\end{tabular}

Block indicates extent of data.

Computed from: $V=9$ for $D^{2} L$ to $48 ; V=0.25248 D^{2} L-3.05798$ for $D^{2} L$ larger than 48

Standard error of estimate: $\pm 21.20 \%$ of mean; \pm 25 board feet

Coefficient of determination: 0.9512

Diameter classes full-inch (e.g., 20-inch class includes 20.0 to 20.9 inches d.b.h.) 
Table 11.-Gross volumes, in board feet inside bark International 1/4-inch Rule per square foot of basal area, merchantable stem excluding stump and top, ponderosa pine in the Front Range of Colorado. Top diameter 6 inches inside bark. Stump height 1 foot

\begin{tabular}{|c|c|c|c|c|c|c|c|c|c|c|}
\hline \multirow[b]{2}{*}{ d.b.h. } & \multicolumn{10}{|c|}{ Number of $16 \cdot$ foot logs to $6 \cdot$ inch top } \\
\hline & 0.5 & 1.0 & 1.5 & 2.0 & 2.5 & 3.0 & 3.5 & 4.0 & 4.5 & 5.0 \\
\hline \multicolumn{11}{|l|}{ inches } \\
\hline 7 & 29 & 36 & 59 & 83 & & & & & & \\
\hline 8 & 23 & 39 & 62 & 85 & 108 & & & & & \\
\hline 9 & 18 & 40 & 63 & 86 & 110 & & & & & \\
\hline 10 & 18 & 41 & 64 & 87 & 111 & & & & & \\
\hline 11 & 19 & 42 & 65 & 88 & 111 & 135 & & & & \\
\hline 12 & 20 & 43 & 66 & 89 & 112 & 135 & 158 & & & \\
\hline 13 & 20 & 43 & 66 & 90 & 113 & 136 & 159 & 182 & & \\
\hline 14 & & 44 & 67 & 90 & 113 & 136 & 159 & 182 & & \\
\hline 15 & & 44 & 67 & 90 & 113 & 137 & 160 & 183 & & \\
\hline 16 & & 44 & 67 & 91 & 114 & 137 & 160 & 183 & 206 & \\
\hline 17 & & 44 & 68 & 91 & 114 & 137 & 160 & 183 & 206 & \\
\hline 18 & & 45 & 68 & 91 & 114 & 137 & 160 & 184 & 207 & \\
\hline 19 & & 45 & 68 & 91 & 114 & 137 & 161 & 184 & 207 & \\
\hline 20 & & 45 & 68 & 91 & 114 & 138 & 161 & 184 & 207 & \\
\hline 21 & & & 68 & 91 & 115 & 138 & 161 & 184 & 207 & \\
\hline 22 & & & 68 & 91 & 115 & 138 & 161 & 184 & 207 & 230 \\
\hline 23 & & & 68 & 92 & 115 & 138 & 161 & 184 & 207 & 230 \\
\hline 24 & & & & 92 & 115 & 138 & 161 & 184 & 207 & 231 \\
\hline 25 & & & & & 115 & 138 & 161 & 184 & 207 & 231 \\
\hline 26 & & & & & 115 & 138 & 161 & 184 & 208 & 231 \\
\hline 27 & & & & & 115 & 138 & 161 & 184 & 208 & 231 \\
\hline 28 & & & & & 115 & 138 & 161 & 184 & 208 & 231 \\
\hline 29 & & & & & 115 & 138 & 161 & 185 & 208 & 231 \\
\hline 30 & & & & & & 138 & 161 & 185 & 208 & 231 \\
\hline
\end{tabular}

Computed from: VIB $=1,650.10451 / D^{2}$ for $D^{2} L$ to $48 ; V / B=46.29093 H-560.66518 / D^{2}$ for $D^{2} L$ larger than 48 Diameter classes full-inch (e.g., 20-inch class includes 20.0 to 20.9 inches d.b.h.) 


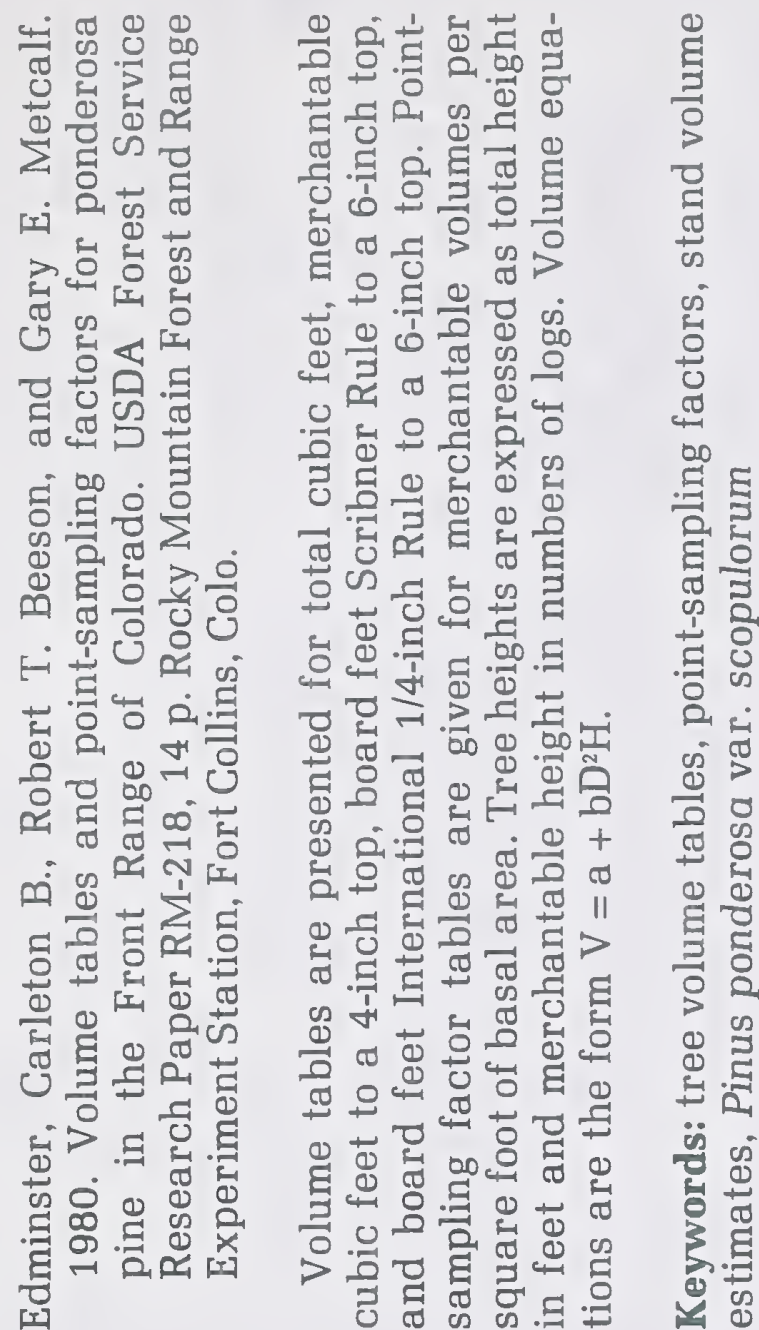

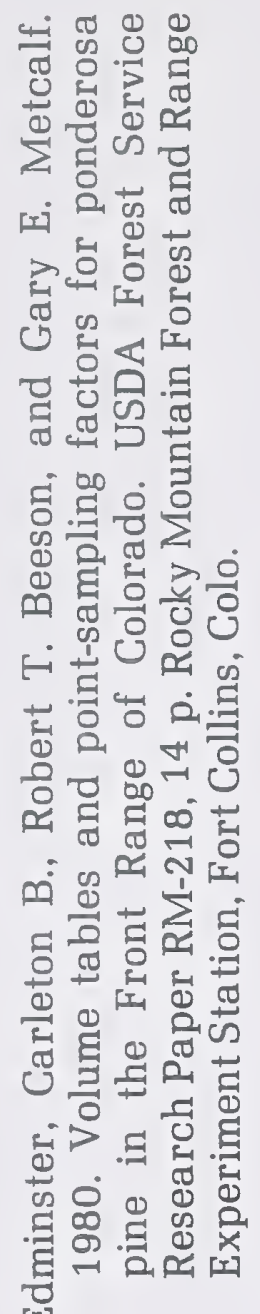

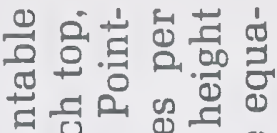

द겅

\%

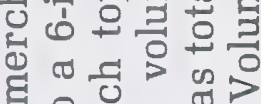

요워

बํำ

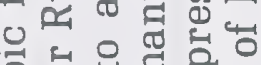

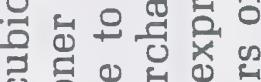

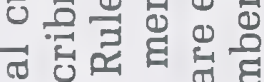

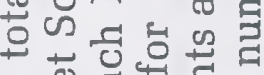

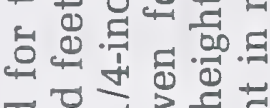

ठ군.

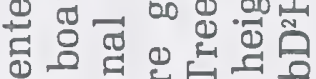

过

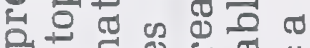

돌

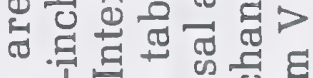

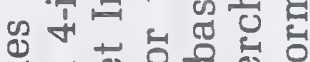

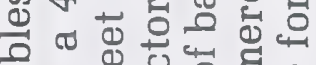

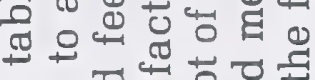

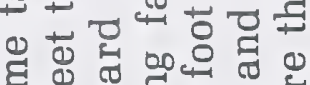

至弯.

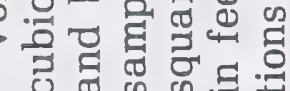

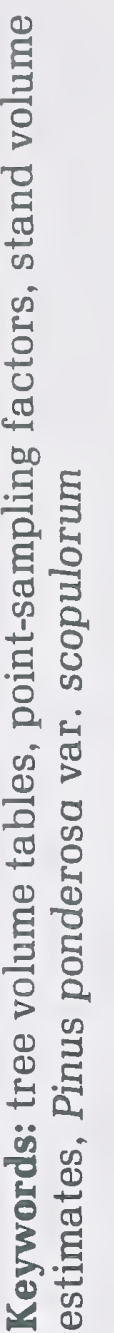

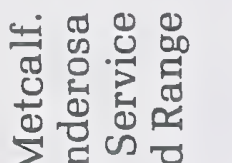

政

되 कू

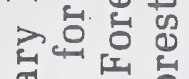

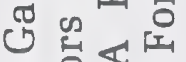

은 동.

สี

ํํํํำ

कำ

纤 흥응

$\therefore$

.

웡

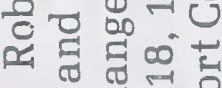

的些理

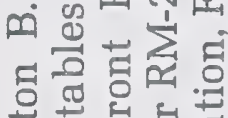

유원

है क

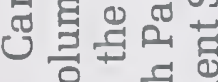

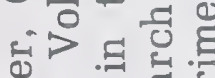

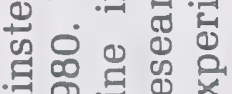

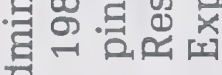

되

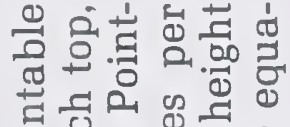

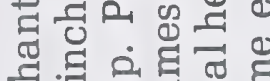

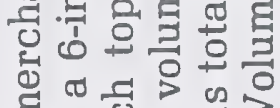

घ귱

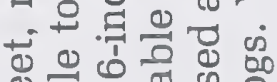

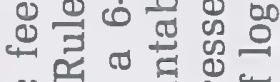

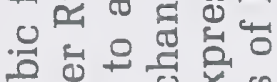

उี

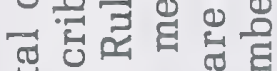

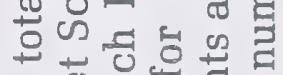

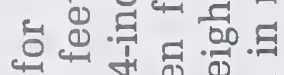

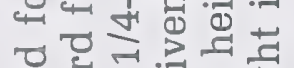

ठ듀.

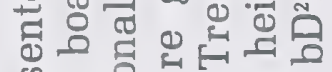

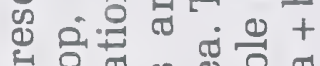

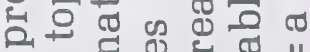

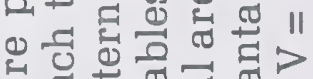

匹.

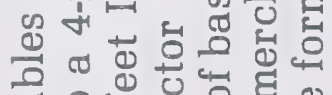

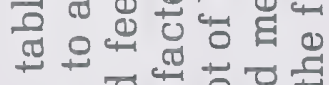

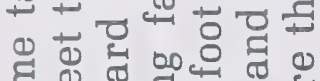

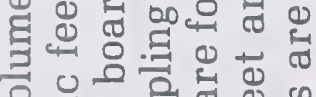

$>00$

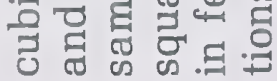

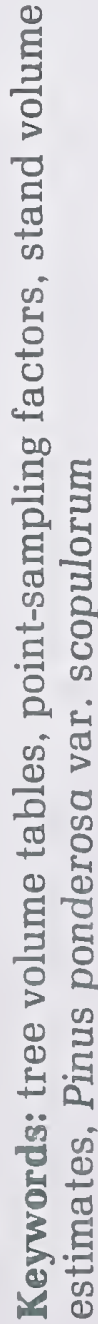

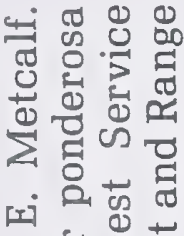

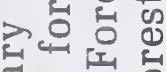

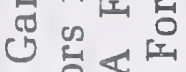

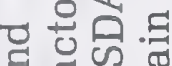

ฮี

¿ี่

कृ 즌

๓

$\rightarrow+0$

E.

远。

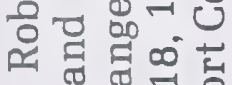
ब $\pi$

صृ

등 范

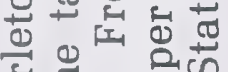

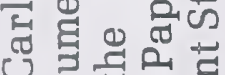

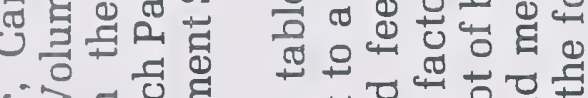

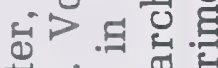

نे

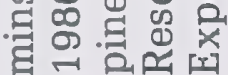
空

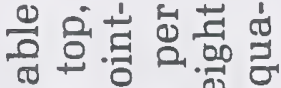

공

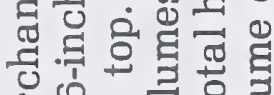

등흐음

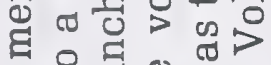

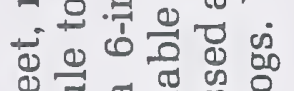

世

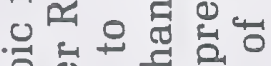

כี

สี

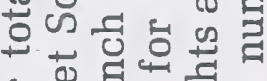

ธุ

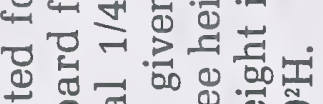

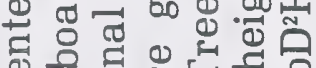

क

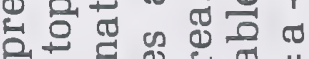

전

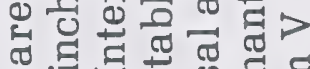

क ने क्षे है

ठ

윰

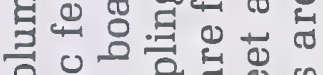

응 웅

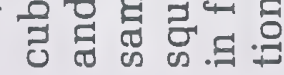

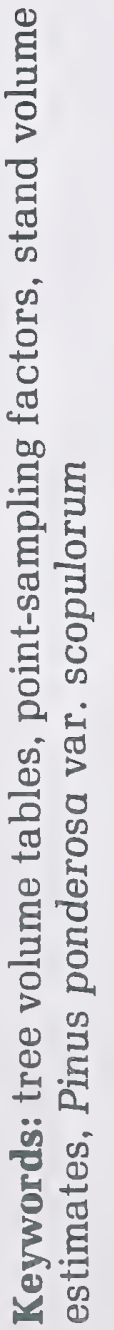




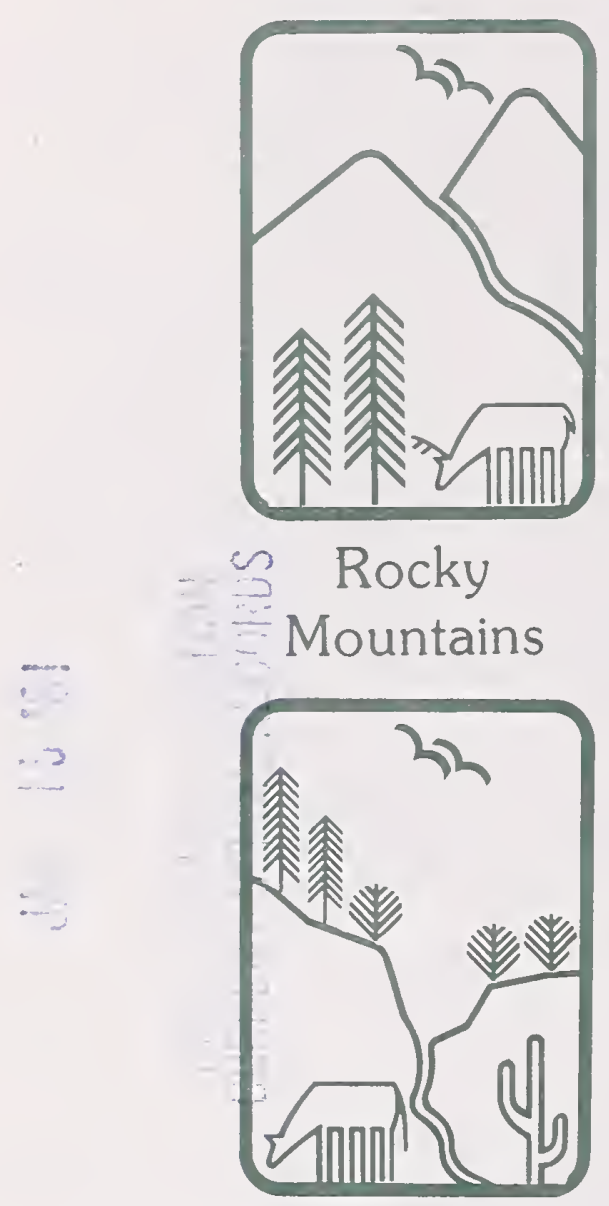

Southwest

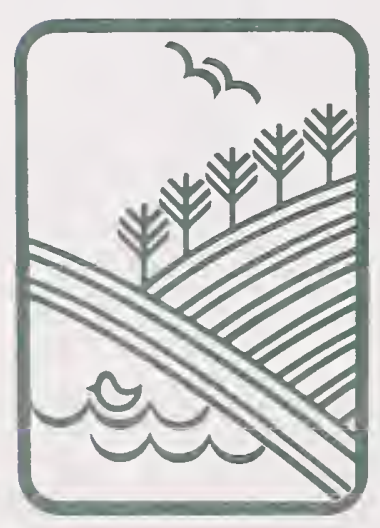

Great Plains
U.S. Department of Agriculture Forest Service

\section{Rocky Mountain Forest and Range Experiment Station}

The Rocky Mountain Station is one of eight regional experiment stations, plus the Forest Products Laboratory and the Washington Office Staff, that make up the Forest Service research organization.

\section{RESEARCH FOCUS}

Research programs at the Rocky Mountain Station are coordinated with area universities and with other institutions. Many studies are conducted on a cooperative basis to accelerate solutions to problems involving range, water, wildlife and fish habitat, human and community development, timber, recreation, protection, and multiresource evaluation.

\section{RESEARCH LOCATIONS}

Research Work Units of the Rocky Mountain Station are operated in cooperation with universities in the following cities:

Albuquerque, New Mexico

Bottineau, North Dakota

Flagstaff, Arizona

Fort Collins, Colorado*

Laramie, Wyoming

Lincoln, Nebraska

Lubbock, Texas

Rapid City, South Dakota

Tempe, Arizona

"Station Headquarters: 240 W. Prospect St., Fort Collins, CO 80526 